\title{
A new model for delivering care for lower urinary tract symptoms
}

Richard Baverstock, $\mathrm{MD}^{1,2}$; R. Trafford Crump, $\mathrm{PhD}^{2}$; Andrea Civitarese, $\mathrm{BSc}^{1}$; Kevin Carlson, $\mathrm{MD}^{1,2}$

${ }^{1}$ vesia [Alberta Bladder Centre], Calgary, AB, Canada; ${ }^{2}$ Department of Surgery, University of Calgary, Calgary, AB, Canada

Cite as: Can Urol Assoc J 2018 November 5; Epub ahead of print. http://dx.doi.org/10.5489/cuaj.5532

Published online November 5, 2018

$* * *$

\section{Abstract}

Introduction: Lower urinary tract symptoms (LUTS) are being treated in secondary care settings, resulting in delayed access for all patients. The objectives of this study were to examine the effects of an integrated delivery model on 1) the volume of care delivered in the secondary care setting; and 2) the use of potentially unnecessary care associated with LUTS.

Methods: This study was based on a retrospective analysis of administrative data collected before and after the integrated LUTS clinic was introduced in Calgary, Alberta. Two cohorts of patients diagnosed with one of four conditions associated with LUTS were defined: 1) the year prior to the introduction of the integrated LUTS clinic; and 2) the year after. To measure their utilization of care, patients' healthcare records between the clinic, emergency department, and hospital were linked. The integrated LUTS clinic involved a multidisciplinary care team, colocated with a common electronic medical record system using a pre-established clinical pathway.

Results: After the introduction of the integrated LUTS clinic, there was a significant increase in the proportional number of patients receiving followup care at the clinic and a significant decrease in the proportional number of patients receiving a cystoscopy or being admitted to the hospital. There was no change in the number of patients visiting the emergency department. Conclusions: An integrated delivery model can be successfully implemented in secondary care for delivering chronic care. The integrated LUTS clinic improved access to care for patients and reduced their use of unnecessary services. 


\section{Introduction}

Integrated delivery models have been advocated as a way to improve the quality of care for patients with chronic illnesses1. While these delivery models have been embraced in many primary care practices, uptake in secondary care settings has not been as widespread due to their complexity2. Yet, increasingly, secondary care settings are caring for patients with chronic illnesses1. This is partly driven by the growing complexity of some chronic conditions and the prevalence of multiple co-morbidities3. It is also driven by some acute conditions being treated more like chronic illness4. Thus, there is a need for rigorous evaluation as to how integrated delivery models perform in secondary care settings.

The focus of this study is the treatment of lower urinary tract symptoms (LUTS). LUTS is defined by storage, voiding, and post micturition symptoms affecting the lower urinary tract5. It is estimated that $13 \%-61 \%$ of the general population has some form of LUTS, with the prevalence increasing to over $83 \%$ in those people aged 75 years or older6,7. These symptoms are often persistent; thus, many conditions associated with LUTS - like overactive bladder, for example - are considered chronic conditions8. Moreover, LUTS is often associated with many comorbid conditions (e.g., high blood pressure, diabetes, depression) and can lead to acute events such as falling9. Despite this, patients presenting to their primary care provider with LUTS, such as overactive bladder, are often referred to urology or gynecology for diagnosis and/or treatment10.

The overall aim of this study is to describe and report on the implementation of an integrated delivery model for caring for LUTS in a secondary care setting. Specifically, the objectives of this study are to examine the effect of this integrated model on the volume of care delivered in the secondary care setting and the provision of potentially unnecessary care associated with LUTS.

\section{Methods}

The vesia delivery model

Faced with the challenges of providing timely and continuous care to patients with LUTS in a secondary care setting, a new clinic was introduced in Calgary, Canada in 2010: vesia [Alberta Bladder Centre]. The clinic was established by two of the authors (RJB, KVC), who are practicing urologists in Calgary with subspecialty fellowships in voiding dysfunction and incontinence.

The model on which the clinic is based shares many of the underlying principles of integrated delivery models. First, it encourages the use of multidisciplinary teams in order to meet the needs of a patient population. The clinical team at vesia includes: specialty-focused nurses, physiotherapists, primary care physicians, internists, gynecologists, and urologists. The team works in a dedicated outpatient clinic, designed specifically for the diagnosis, testing, and treatment of lower urinary tract conditions. 
Second, all providers follow identical guidelines-based clinical care pathways, with appropriate indicators established to identify patients needing to be transitioned to other providers. "Red flags" are embedded into the central intake and clinical care pathways to identify patient problems that require more immediate sub-specialty care or investigation (e.g., hematuria or prior incontinence surgery).

Third, care is shared between providers deemed to be the most appropriate for the patient at that time. For example, a patient may be seen initially by a primary care physician, but later have urodynamic testing with a nurse, and then possibly seen by a specialist for an opinion regarding surgical intervention (e.g., onabotulinumtoxinA injection). Alternatively, a patient referred with overactive bladder and with documented microhematuria (i.e., a "red flag") would initially see a urologist, but then transition as necessary, for example, to physiotherapy and/or primary care for follow-up on pelvic floor muscle training or medication.

This care is coordinated using a common electronic medical record (EMR) system, which all providers have access to. The clinic is physically laid out to encourage discussion and communication amongst providers, with a central computer station between all the assessment and treatment rooms, and clinics are run in parallel to ensure that each discipline is present at any given time. Finally, all the providers use common patient-education materials, ensuring that a consistent message is delivered to patients regardless of which provider is delivering their care.

\section{Study design}

In order to evaluate the effect of the new vesia clinic, a retrospective study was undertaken. Two cohorts of patients with LUTS were defined for this study. The first represents all new patients seen by the two urologists (RJB, KVC) in the year prior to establishing the new clinic (i.e., January 1, 2010 to December 31, 2010). The second cohort represents all new patients seen using the new delivery model in the year after the vesia clinic was established (i.e., January 1, 2012 to December 31, 2012). Data for both cohorts was extracted from the EMR. Patients were identified if they had an International Classification of Diseases, Ninth Revision (ICD-9) code of 600 (benign prostatic hypertrophy), 788.2* (retention of urine), 788.3* (urinary incontinence), and 788.4* (frequency of urination and polyuria). We selected these codes over others because they represented those that were used with the most frequency.

Both cohorts were linked with administrative health care utilization data from Alberta Health Services, the health authority in the province. Linking was done using patients' first and last name, date of birth, and their personal health number - a unique identifier issued to every resident in the province. These administrative data included all health care services used by the patients, regardless of whether or not it was related to LUTS. Alberta Health Services uses ICD10 codes; primary, secondary, and tertiary diagnostic codes were used to identify use of health care services that may be related to the patient's LUTS. We identified those codes in the ICD-10 $\mathrm{N}$ range (i.e., "diseases of the genitourinary system") that could be reasonably used to classify a 
patient's LUTS at the time of hospital admission or emergency department visit. All other visits were dropped from the analysis. The administrative data also contained a pre-calculated Charlson Comorbidity Index11, a weighted index to classify a patient's current comorbid conditions.

The volume of care was defined as the number of clinical encounters with patients diagnosed with LUTS in the secondary care setting. It was operationally measured by the unique number of patients and the number of visits. Visits were further characterized by: 1) the type of provider that was seen, and 2) whether it was an initial consult or follow-up. Encounters with physiotherapists, while part of the integrated vesia delivery model, were not included in the analysis as these types of encounters are not available in the administrative data.

The provision of potentially unnecessary care associated with LUTS was defined as health care services that, if the underlying condition were being managed effectively, should not occur. It was operationally measured by the use of: 1) the number of cystoscopies, 2) visits to the emergency department; and, 3) inpatient hospitalizations.

Given the retrospective nature of this study, a waiver of consent was approved by the University of Calgary's Conjoint Health Research Ethics Board. All data were de-identified prior to analysis using Stata version 14 (StataCorp LP, College Station, TX).

\section{Data analysis}

Descriptive statistics were used to characterize the two cohorts in terms of their demographic and clinical variables using EMR data from vesia. Comparisons included age, sex, and type of visit (i.e., initial or follow-up). T-tests or Pearson's chi2 were used to test for significant differences between the two cohorts. The level of statistical significance was defined as 0.05.

The administrative data were separated into three groups: cystoscopy, emergency department visits, and hospital discharges. Within each group, the number of unique patients was identified. Two-by-two tables were constructed comparing the number of unique patients who incurred a visit versus those who did not. The number of patients who did not incur a visit was calculated by taking the total number of unique patients in each yearly cohort minus the number of patients who experienced a visit. Pearson chi2 was calculated, along with odds ratios. Additionally, average number of visits per patient were calculated and compared using t-tests.

\section{Results}

In the 2010 pre-vesia cohort, 744 new patients with LUTS were seen by the two urologists in their general urology practices. In 2012, the year following implementation of the integrated model, 1,457 patients were seen by the multidisciplinary team of care providers. As detailed in Table 1, there were statistically significant differences between the two cohorts. The 2012 cohort had a median age that was comparatively lower to that from 2010, with a larger range of age. As a proportion of the overall sample, the 2012 cohort also was significantly more female. The mix 
of diagnoses also changed significantly between the two years, with proportionally more "frequency of urination and polyuria" (i.e., 788.4) being diagnosed in 2012.

Change in the volume of LUTS care

Patients in the 2010 cohort had a total of 994 visits to the two urologists, compared to 2,062 visits to the vesia clinic in 2012. Patients received an average of 1.36 visits in 2010, compared to 1.42 visits in 2012. As detailed in Table 1, the proportional make up of these visits changed with the introduction of the vesia clinic. In 2010, initial visits represented $75 \%$ of all visits, compared to $71 \%$ in 2012. Consequently, there were more follow-up visits in 2012 compared to 2010. The change in proportional make up of visits was statistically significant (Pearson chi2 $=5.84, \mathrm{p}=$ 0.015).

In 2010, all new and follow-up visits were conducted by the two urologists. In 2012, 63\% of the new visits and $54 \%$ of the follow-up visits (data not shown) were conducted by primary care physicians at the vesia clinic. Urologists and gynecologists conducted the remaining new and follow-up patient visits, and urologists conducted all of the cystoscopies.

Reduction in unnecessary care

Use of cystoscopy

In 2010, there were 491 (66\%) patients diagnosed with LUTS that received a cystoscopy. In 2012, this increased to on an absolute basis $(n=638)$, but decreased as a proportion of all patients (44\%), see Table 2 . The proportional difference between the two cohorts was statistically significant (Pearson chi2 $=97.2, \mathrm{p}<0.0001$ ).

The mean age of patients undergoing a cystoscopy decreased from 63.9 to 63.1 years between 2010 and 2012. There was also an increase from 2010 to 2012 in the percentage of females receiving this service, from $26 \%$ to $38 \%$. The mean Charlson Comorbidity Index changed from $0.17(\mathrm{SD}=0.51)$ to 0.15 ( $\mathrm{SD}=0.64$ ), indicating a slight reduction in the complexity of patients. The changes in mean age and Charlson Comorbidity Index were not statistically significant, however the change in the distribution of gender was (Pearson chi2 = 29.6, $\mathrm{p}<0.001$ ).

Use of the emergency department

There were 8 (1\%) patients from the 2010 cohort who sought care from the emergency department, compared to 31 (2\%) patients from the 2012 cohorts. The change in the proportional distribution of patients seeking emergency care was not statistically significant (Pearson chi2 $=3.13, \mathrm{p}=0.076$ ), as detailed in Table 3 .

The mean age of patients accessing these services decreased from 64.8 to 60.1 years between 2010 and 2012. The mean Charlson Comorbidity Index changed from 0.01 (SD < 0.001 ) to 0.25 ( $\mathrm{SD}=0.65$ ), indicating more complex patients sought care from the emergency 
department in 2012 as compared to 2010. None of the changes between the yearly cohorts were statistically significant.

Use of the hospital

Ninety-one patients were admitted to hospital as an inpatient in the 2010 cohort, compared to 119 patients in the 2012 cohort. As detailed in Table 4, proportionally fewer patients were treated as an inpatient in 2012 compared to 2010 (8\% in 2012 compared to 12\% in 2010); this difference was statistically significant (Pearson chi2 $=9.42, \mathrm{p}=0.002$ ).

The mean age of inpatients increased from 70.8 to 71.6 years between 2010 and 2012. The mean Charlson Comorbidity Index changed from $0.68(\mathrm{SD}=1.10)$ in 2010 to 0.89 (SD = 1.96) in 2012. Neither the change in mean age nor in the Charlson Comorbidity Index were statistically significant.

\section{Discussion}

With the demand for chronic care services from secondary care far outpacing the supply of medical specialists available to treat them, access to, and the quality of, care for chronic conditions is under pressure. New delivery models in secondary care settings are needed to more effectively address the needs of patients with chronic illnesses. This study demonstrates that an integrated delivery model for chronic care, long advocated as an exemplar in primary care, can be equally successful in a secondary care setting. The focus of this study was LUTS, representing a set of conditions that are increasingly being treated by urologists and gynecologists in their practices. Results from this retrospective analysis of a multidisciplinary outpatient clinic focused on the treatment of lower urinary tract conditions suggest that an integrated delivery model provides significant benefit to the health care system.

The integrated delivery model significantly increased the volume of LUTS-related care delivered in the secondary care setting. Not only were more patients with LUTS seen after the new vesia model was introduced - as would be expected with an expanded staff of care providers - but the average number of visits these patients were provided also increased. In other words, patients received significantly more follow-up care under the vesia model. After the vesia clinic was introduced, there was a significant reduction in the proportional number of patients who received a cystoscopy, a potentially unnecessary intervention for patients with LUTS. There was also a significant decrease in the proportional number of hospitalizations for any LUTS-related diagnosis after vesia was introduced.

The observations from this study concur with the few others that have investigated the use of integrated delivery models in outpatient, secondary care settings. In cardiovascular care, the introduction of multidisciplinary teams has resulted in a significant reduction in length of hospital stays between 36\% - 54\%, hospital readmission rates between 42\% - 62\%, and cost per patient12. Cancer care has also embraced the multidisciplinary approach leading to decreased time from diagnosis to treatment, lower costs, and better treatment outcomes13. 
Beyond the direct benefit to patients with overactive bladder, the use of an integrated delivery model has a positive impact on a broader patient base. When specialists are asked to care for patients with chronic conditions like overactive bladder, there is the chance that they may crowd-out patients with more urgent needs with potentially debilitating or life-threatening conditions. By incorporating an integrated model, like the vesia clinic, specialists have more availability to assess and care for these other patients.

The generalizability of this study is limited by several factors. First, this study evaluates a single center, which may have some unique characteristics that influenced the results. A multicenter study would allow for the potential inherent biases to be controlled. However, it was not possible to coordinate such an effort across Alberta's health care system. A related limitation is that patients were not randomized between the conventional and integrated delivery models. There is the potential that physicians selectively referred their patients to the vesia clinic, unduly influencing the results in some manner. Indeed, our analyses indicate that the 2010 and 2012 cohorts evaluated in this study differed in terms of their age, gender, and condition. A few surprising findings came forward in this analysis that warrant discussion. The age decrease is minimal and still shows that the majority of LUTS patients are older. However, the increase in female patients likely came from increased awareness of the vesia clinic in the community and a shift in referrals from the government-run pelvic floor clinic. It may also be explained by a triage of female LUTS patients from the other Calgary urologists so they could enjoy a subspecialization away from overactive bladder (OAB) and LUTS. Likewise, the proportion of patients diagnosed with "Frequency of urination and polyuria" moved from $15-52 \%$ which came from a re-direction of both female and male patients with this diagnosis from the local urologists as well as a growing awareness of the vesia clinic in the referral community. Finally, patientreported outcomes and patient experience with the delivery models were not measured. Thus, no observations can be made regarding patients' perspectives of the change in delivery models. The desire to create a multi-disciplinary model was born from the frustration of being overwhelmed with LUTS referrals that prevented the urologists (RJB and KVC) from seeing surgical patients. It was not unusual to see 30-40 patients in a clinic and book no surgeries because primary $\mathrm{OAB}$ or recurrent urinary tract infection referrals filled the clinics of the urologists. However, to successfully implement a model like vesia [Alberta Bladder Centre], a team must be assembled who have a common goal of delivering LUTS care in an expedited and similar fashion. Pathways should be established so that practitioners are interchangeable to a large degree but also complimentary for example a primary care physician may assess a patient for OAB but suggest a pessary with the clinic nurse and a pelvic floor physiotherapy session with the team's physiotherapist. All care is delivered in-house, facilitating the use of the EMR as a central hub to coordinate care internally. As well, the urologist is ultimately the most responsible physician who is messaged or involved for red flags such as hematuria or consultation for surgical therapy. 


\section{Conclusion}

An integrated delivery model can be successfully implemented in secondary care for delivering chronic care. This model can improve access to care for patients and potentially reduce their use of unnecessary services. Integrated models, like the vesia clinic in this study, should be considered by other medical specialists faced with treating an increasing number of chronically ill patients in a secondary care setting. 


\section{References}

1. Wagner EH, Austin BT, Davis C, et al. Improving Chronic Illness Care: Translating Evidence Into Action. Health Aff 2001; 20(6), 64-78.

2. Johnson C. Health Care Transitions: A Review of Integrated, Integrative, and Integration Concepts. J Manipulative Physiol Ther 2009;32:703-713.

3. Chwastiak L, Vanderlip E, Katon W. Treating complexity: Collaborative care for multiple chronic conditions. Inter Rev Psychiatry 2014;26:638-647.

4. Rothman AA, Wagner EH. Chronic Illness Management: What Is the Role of Primary Care? Ann Intern Med 2003;138:256.

5. Abrams P, Cardozo L, Fall M, et al. The standardisation of terminology of lower urinary tract function: report from the Standardisation Sub-committee of the International Continence Society. Neurourol Urodyn 2002;21: 167-78

6. Irwin DE, Kopp ZS, Agatep B, et al. Worldwide prevalence estimates of lower urinary tract symptoms, overactive bladder, urinary incontinence and bladder outlet obstruction. BJU Int 2011;108:1132-1138.

7. Irwin D, Milsom I, Reilly K, et al. Prevalence of lower urinary tract symptoms (LUTS) in men: European results from the EPIC study Eur Urol Suppl 2006;5:157-157.

8. Coyne KS, Thompson CL, Lai J, et al. An overactive bladder symptom and health-related quality of life short-form: Validation of the OAB-q SF. Neurourol Urodyn 2015;34:255263.

9. Tannenbaum C, Gray M, Hoffstetter S, et al. Comorbidities associated with bladder dysfunction. Int J Clin Pract 2013;67:105-113.

10. Nguyen K, Hunter KF, Wagg A. Knowledge and understanding of urinary incontinence Survey of family practitioners in northern Alberta. Can Fam Physician 2013;59:e330.

11. Charlson ME, Pompei P, Ales KL, et al. A new method of classifying prognostic comorbidity in longitudinal studies: development and validation. J Chron Dis 1987;40(5), 373-83. Retrieved from http://www.ncbi.nlm.nih.gov/pubmed/3558716

12. Balinsky W, \& Muennig P. The costs and outcomes of multifaceted interventions designed to improve the care of congestive heart failure in the inpatient setting: a review of the literature. Med Care Res Rev 2003. doi:10.1177/1077558703254697

13. Abramson JS, \& Mizrahi T. When social workers and physicians collaborate: positive and negative interdisciplinary experiences. Soc Work 1996;41(3), 270-281 
Figures and Tables

\begin{tabular}{|c|c|c|c|}
\hline & $\begin{array}{l}2010 \\
\text { n (\%) }\end{array}$ & $\begin{array}{l}2012 \\
\text { n (\%) }\end{array}$ & \\
\hline $\mathrm{n}$ & 744 & 1,457 & \\
\hline Total visits & 994 & 2,062 & \\
\hline Initial visits & $744(75)$ & $1,457(71)$ & Pearson $\mathrm{Chi}^{2}=5.84$ \\
\hline Followup visits & $250(25)$ & $605(29)$ & $\mathrm{p}=0.015$ \\
\hline \multicolumn{4}{|l|}{ Age at first visit } \\
\hline Median years & 65 & 62 & $t=3.85$ \\
\hline Min-max years & $18-96$ & $17-98$ & $p<0.001$ \\
\hline Interquartile range & 23 & 24 & \\
\hline Standard deviation & 16.0 & 16.7 & \\
\hline \multicolumn{4}{|l|}{ Gender } \\
\hline Male & $548(74)$ & $712(49)$ & Pearson $\mathrm{Chi}^{2}=123.6$ \\
\hline Female & $196(26)$ & 745 (51) & $\mathrm{p}<0.001$ \\
\hline \multicolumn{4}{|l|}{ Diagnosis } \\
\hline Benign prostatic hypertrophy & $193(26)$ & 248 (17) & Pearson $\mathrm{Chi}^{2}=408.7$ \\
\hline Retention of urine & $191(26)$ & 348 (24) & $\mathrm{p}<0.001$ \\
\hline Urinary incontinence & 249 (33) & $102(7)$ & \\
\hline $\begin{array}{l}\text { Frequency of urination and } \\
\text { polyuria }\end{array}$ & $111(15)$ & 759 (52) & \\
\hline \multicolumn{4}{|l|}{ Physician-type at first visit } \\
\hline Urology & $744(100)$ & 912 (63) & \\
\hline GP & -- & $529(36)$ & \\
\hline Gynecology & -- & $16(1)$ & \\
\hline
\end{tabular}




\begin{tabular}{|l|c|c|c|}
\hline \multicolumn{3}{|c|}{ Table 2. Comparison of patients undergoing a cystoscopy between yearly cohorts } \\
\hline & \multicolumn{2}{|c|}{ Patients undergoing a cystoscopy } \\
& Y\%) & No & Total \\
\hline \multirow{2}{*}{2010} & 491 & 253 & 744 \\
& $(66.0)$ & $(34.0)$ & 1457 \\
& 638 & 819 & \\
\hline
\end{tabular}

Pearson $\mathrm{Chi}^{2}=97.21 ; \mathrm{p}<0.001$. Odds ratio 2.49 (95\% confidence interval 2.06-3.01).

\begin{tabular}{|c|c|c|c|}
\hline & Patients visit & y department & \\
\hline & Yes & No & Total \\
\hline 2010 & $\begin{array}{c}8 \\
(1.1)\end{array}$ & $\begin{array}{c}736 \\
(98.9)\end{array}$ & 744 \\
\hline 2012 & $\begin{array}{c}31 \\
(2.1)\end{array}$ & $\begin{array}{l}1,426 \\
(97.9)\end{array}$ & 1457 \\
\hline
\end{tabular}

Pearson $\mathrm{Chi}^{2}=3.13 ; \mathrm{p}=0.076$. Odds ratio 2.00 (95\% confidence interval 0.89-5.06).

\begin{tabular}{|c|c|c|c|}
\hline & Patients wi & pitalizations & \\
\hline 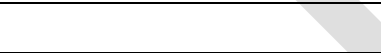 & Yes & No & Total \\
\hline 2010 & $\begin{array}{c}91 \\
(12.2)\end{array}$ & $\begin{array}{c}653 \\
(87.8)\end{array}$ & 744 \\
\hline 2012 & $\begin{array}{l}119 \\
(8.2)\end{array}$ & $\begin{array}{l}1,338 \\
(91.8)\end{array}$ & 1457 \\
\hline
\end{tabular}

Pearson $\mathrm{Chi}^{2}=9.42 ; \mathrm{p}=0.002$. Odds ratio 0.64 (95\% confidence interval $0.47-0.86$ ). 